\title{
BMJ Open Are patients' goals in treatment associated with expected treatment outcomes? Findings from a mixed- methods study on outpatient pharmacological treatment for opioid use disorder
}

\author{
Tea Rosic (1) , ${ }^{1,2}$ Leen Naji (1) , ${ }^{2,3}$ Balpreet Panesar, ${ }^{4}$ Darren B Chai, ${ }^{5}$ \\ Nitika Sanger (D) , ${ }^{6}$ Brittany B Dennis, ${ }^{7}$ David C Marsh, ${ }^{8,9,10,11}$ Launette Rieb, ${ }^{12}$ \\ Andrew Worster, ${ }^{2,7}$ Lehana Thabane, ${ }^{2,13}$ Zainab Samaan (D) ${ }^{1,2}$
}

To cite: Rosic T, Naji L, Panesar B, et al. Are patients' goals in treatment associated with expected treatment outcomes? Findings from a mixed-methods study on outpatient pharmacological treatment for opioid use disorder. BMJ Open 2021;11:e044017. doi:10.1136/ bmjopen-2020-044017

\section{- Prepublication history and} additional material for this paper is available online. To view these files, please visit the journal online (http://dx.doi.org/10. 1136/bmjopen-2020-044017)

Received 21 August 2020 Revised 11 December 2020 Accepted 15 December 2020

Check for updates

(C) Author(s) (or their employer(s)) 2021. Re-use permitted under CC BY-NC. No commercial re-use. See rights and permissions. Published by BMJ.

For numbered affiliations see end of article.

Correspondence to

Dr Zainab Samaan;

samaanz@mcmaster.ca

\section{ABSTRACT}

Objectives Existing methods of measuring effectiveness of pharmacological treatment for opioid use disorder (OUD) are highly variable. Therefore, understanding patients' treatment goals is an integral part of patient-centred care. Our objective is to explore whether patients' treatment goals align with a frequently used clinical outcome, opioid abstinence.

Design Triangulation mixed-methods design.

Setting and participants We collected prospective data from 2030 participants who were receiving methadone or buprenorphine-naloxone treatment for a diagnosis of OUD in order to meet study inclusion criteria. Participants were recruited from 45 centrally-managed outpatient opioid agonist therapy clinics in Ontario, Canada. At study entry, we asked, 'What are your goals in treatment?' and used NVivo software to identify common themes.

Primary outcome measure Urine drug screens (UDS) were collected for 3 months post-study enrolment in order to identify abstinence versus ongoing opioid use (mean number of UDS over 3 months=12.6, SD=5.3). We used logistic regression to examine the association between treatment goals and opioid abstinence.

Results Participants had a mean age of 39.2 years $(\mathrm{SD}=10.7), 44 \%$ were women and median duration in treatment was 2.6 years (IQR 5.2). Six overarching goals were identified from patient responses, including 'stop or taper off of treatment' (68\%), 'stay or get clean' $(37 \%)$ and 'live a normal life' (14\%). Participants reporting the goal 'stay or get clean' had lower odds of abstinence at 3 months than those who did not report this goal $(\mathrm{OR}=0.73$, $95 \% \mathrm{Cl} 0.59$ to $0.91, p=0.005)$. Although the majority of patients wanted to taper off or stop medication, this goal was not associated with opioid abstinence, nor were any of their other goals.

Conclusions Patient goals in OUD treatment do not appear to be associated with programme measures of outcome (ie, abstinence from opioids). Future studies are needed to examine outcomes related to patient-reported treatment goals found in our study; pain management,
Strengths and limitations of this study

- This study is strengthened by its large sample size (2000 participants) and multisite design.

- Participating clinics follow a harm-reduction approach to treatment and these findings may not generalise to abstinence-based treatment settings.

- The goals and treatment outcomes of patients newly entering treatment may differ from those of patients who have been in treatment longer and may not be captured in this study.

employment, and stopping/tapering treatment should all be explored.

\section{INTRODUCTION}

Opioid use disorder (OUD) remains a clinical and public health challenge, with ongoing high rates of opioid use and overdose deaths. ${ }^{1}$ Consequently, growing numbers of patients are enrolled in pharmacological treatment for OUD. ${ }^{23}$ Methadone, a full opioid agonist, and buprenorphine, a partial opioid agonist, are the two most commonly used medications in the management of OUD; they act to reduce cravings and withdrawal, and support abstinence from ongoing opioid use. ${ }^{4}$ Evidence from systematic reviews of experimental studies indicates that both medications reduce opioid use. ${ }^{56}$ However, not all patients have favourable outcomes, ${ }^{7}$ and patients who continue to use opioids during treatment have a high risk of overdose and death. ${ }^{9} 10$ Other treatments, including heroin-assisted treatment, are available in 
some jurisdictions for patients who have limited response to treatment with first-line medications. ${ }^{11}$

Better understanding patients' goals in treatment is considered increasingly important within the field of substance use and addiction. ${ }^{12-14}$ The now well-known concept of patient-centred care was originally coined with the definition of "care that is respectful of, and responsive to, individual patient preferences, needs and values", ${ }^{1516}$ and is demonstrated to have a significant impact on patients' outcomes and satisfaction in treatment. ${ }^{17}$ Increasing attention is being paid to patients' goals and the implementation of patient-centred care principles in addiction treatment. $^{18}$

Identifying core treatment outcomes is an active area of investigation within the field of Addiction Medicine. ${ }^{19}$ Unfortunately, there is still significant variability in the outcomes used to evaluate the effectiveness of pharmacological treatment for OUD. ${ }^{20}{ }^{21}$ How to best measure and assess treatment outcomes remains uncertain, and current practices risk being based on convenience. Opioid use, measured by urine drug screens (UDS), and retention in treatment are the most commonly used primary outcomes measured in clinical studies and treatment programmes; ${ }^{21}$ however, it is unknown how well these outcomes are associated with patients' goals in treatment. Personal and social functioning outcomes are, in contrast, much less commonly assessed. ${ }^{21}$ As core endpoints and outcome sets for studies of OUD are developed, it is critical to understand which goals in treatment are important to patients and how to best measure them.

In a recent study by Sanger et al, 2020, we used qualitative analysis methods to examine patient-reported treatment goals in a cohort of more than 2000 patients receiving outpatient pharmacological treatment for OUD. ${ }^{22}$ We identified six distinct goals in treatment from patient responses, including to control cravings or withdrawal, to maintain or stabilise medication dose, to stop or taper off treatment, to 'stay or get clean', to manage pain and to 'live a normal life'.22

The objective of the present study was to explore whether these patient-reported treatment goals are associated with abstinence from opioid use (a frequently measured programme outcome). We hypothesised that patient goals related to drug use would be associated with opioid use during treatment; meanwhile, goals unrelated to drug use would have no association with UDS results.

\section{METHODS \\ Data}

We collected prospective observational data from 2030 participants recruited from 45 outpatient clinics in the Pharmacogenetics of Opioid Substitution Treatment Response (POST) study. To meet study inclusion criteria, participants were required to be at least 16 years of age and receive pharmacological treatment with methadone or buprenorphine-naloxone (for any length of time) for a diagnosis of OUD, as per the Diagnostic and Statistical
Manual of Mental Disorders, $5^{\text {th }}$ Edition (DSM-5). ${ }^{23}$ The diagnosis of OUD was made by treating physicians according to DSM-5 criteria and is an eligibility criterion for treatment entry and clinical follow-up at the outpatient clinics included in this study. No other inclusion or exclusion criteria were applied in order to increase the generalisability of this study. Participants completed faceto-face interviews at study entry to collect information on demographic and clinical characteristics.

We used a triangulation mixed-methods design to combine quantitative and qualitative data collection, where both quantitative and qualitative data were collected within one study instrument using closed- and open-ended questions. ${ }^{24}{ }^{25}$ At study intake, participants were interviewed by trained research staff to obtain information on sociodemographic and clinical information, medical history and substance use history. Research staff had a background in addiction research, as they previously participated in recruitment of participants for a study investigating genetic influences on methadone treatment. ${ }^{26}$ Their experience allowed for familiarity of addiction-related terms used in interview responses; however, research staff were not known to the participants of this study. Study interviews were conducted in-person. The interview data used in this study is from participants recruited between May 2018 and August 2019. During the interview, all participants who met the inclusion and exclusion criteria above were asked the open-ended question, 'What are your goals in treatment?'. Details regarding the study settings and data collection are outlined in our previous study. ${ }^{22}$ Verbal responses, in their entirety, were transcribed by research staff word-for-word in online anonymised records, where each participant was given an anonymised record number.

We collected the results of UDSs for opioids for each participant for 3 months following study entry to assess treatment outcome. The FaStep Assay (Trimedic Supply Network Ltd, Concord, Ontario, Canada) was used to detect morphine, oxycodone, fentanyl, methadone metabolite and buprenorphine, as well as other non-opioid substances. ${ }^{27}$ Though other methods may be used to assess ongoing opioid use during treatment, such as saliva and hair tests, as well as self-reported drug use, ${ }^{28}$ UDSs are collected as part of routine clinical protocol in the clinics participating in this study and are a recommended method of assessment based on Canadian Guidelines. ${ }^{4}$ UDSs were collected following clinic protocol (typically weekly or biweekly). For each participant, we calculated the percentage of opioid-positive UDSs by dividing the number of opioid-positive urines by the number of urine samples taken. Abstinence from opioids was selected as our primary study outcome as it is a routinely measured treatment outcome in both clinical practice and research studies.

Another commonly studied treatment outcome, retention in treatment, was not formally assessed in the present study for two reasons. First, treatment retention is not equivalent to the duration of time enrolled in this study 
(as our study used a naturalistic design and enrolled patients in various stages of their treatment). Second, with the exception of patients who have entered treatment for the first time, there exists some uncertainty in defining treatment retention because patients frequently enter and discontinue treatment at various points in their course of illness. Instead, we asked participants to report their length of time enrolled in this treatment episode (as a proxy for treatment retention) and adjusted all study analyses for length of time in treatment.

We report methods and quantitative results in accordance with the Strengthening the Reporting of Observational Studies in Epidemiology (STROBE) guidelines. ${ }^{29}$

\section{Qualitative analysis}

The qualitative approach used to analyse the data was data-driven thematic analysis. ${ }^{30}$ We began by familiarising ourselves with the data through active, repeated reading of the interview responses and began to recognise emerging patterns. This phase of data familiarisation also allowed us to minimise typographical errors present in the free-text responses. We began phase two by generating initial codes using NVivo software (QSR International (Americas) Inc, Burlington, Massachusetts, USA) for qualitative analysis to identify common themes from patient answers. ${ }^{31}$ We began cataloguing main ideas, phrases and patterns into meaningful nodes using word and text queries, and a review of the transcribed data. Word and text queries helped us capture the patterns in data and improve analytical accuracy by identifying stemmed variants. Each data item was given equal attention and in addition to text and word queries, key phrases were tagged within each data item. This phase is characterised by the generation of a codebook that provided specific definitions of the key phrases, words and patterns. The next phase consisted of the labelling of some nodes as themes and the collapsing of related nodes into one node, eventually being labelled as a themes or subthemes. The final phase consisted of a review of identified themes and resultant reworking of themes to better establish coherent patterns within each theme. Defining and refining of each theme followed this phase, where patterns and content were considered before choosing relevant and reflective theme names. ${ }^{30} 32$ To increase rigour in our analysis, we used investigator triangulation, where phases concerning the generation of themes involved the consultation of four investigators to ensure incorporation of diverse perspectives. This was reflected in the iterative review of nodes and patterns, where meaningfulness of coding was discussed and was reassessed at every identified phase. We report qualitative methods and results in accordance with the Standards for Reporting Qualitative Research (SRQR). ${ }^{33}$

\section{Quantitative analysis}

We conducted all quantitative analyses using Stata version 15.1 (StataCorp LP, College Station, Texas, USA). We report demographic and clinical data using mean and SD for normally distributed continuous variables and median with quartiles 1 and 3 or interquartile range (IQR) for skewed data. We report categorical variables as frequency with percentage. We summarise the results of UDSs in three ways: (1) the mean number of UDSs collected; (2) the percentage of opioid-positive UDSs; and (3) abstinence from opioid use, defined as no opioid-positive UDSs during the 3-month time period.

We used logistic regression analysis to examine the association between patient goals in treatment and abstinence from opioid use, adjusting for other important covariates. We constructed a logistic regression model, using the dependent variable abstinence from opioid use throughout the 3 months following study entry. We included the six identified treatment goals in the model and controlled for other factors believed to impact ongoing opioid use in treatment, including age, sex ${ }^{34}{ }^{35}$ type of treatment (methadone or buprenorphine-naloxone), medication dose, ${ }^{36}$ length of time in treatment ${ }^{37}$ and abstinence from opioids at baseline. We also conducted an additional logistic regression to determine whether the number of goals reported by participants was associated with opioid abstinence, as patients who report more treatment needs tend to have more opioid use. ${ }^{38}$ Results are reported as ORs with 95\% CIs and associated p values. We report the estimates of effect for our main variables of interest (treatment goals) in the results table and describe all variables adjusted for in a footnote in the table in order to focus solely on the variables of interest to our specific study question. We assessed for multicollinearity using variance inflation factor (VIF) and examined model diagnostics using the Hosmer-Lemeshow statistic and deviance residuals. We conducted a sensitivity analysis after excluding observations with a deviance residual lower than -2 or higher than 2 . Our sample size of 2030 participants and event rate of more than 1000 participants abstinent from opioids is adequate, based on the rule of thumb for number of events needed $(n=10)$ per covariate included in logistic regression analysis. ${ }^{39}$

Missing data were identified and reported for each variable of interest. There were less than five cases with missing data for baseline demographic or clinical variables. For 3-month UDS, missing data affected 34 participants (1.7\%). Reasons for missing 3-month UDS data included: results not yet available $(n=6)$, transfer to another clinic $(n=8)$, treatment failure $(n=10)$, incarceration $(n=3)$, completion of treatment $(n=2)$ and other $(n=4)$, such as hospitalisation, moving or never starting treatment. Due to the low percentage of missing data, all missing data were handled by available case analysis.

\section{Patient and public involvement}

Patients or the public were not involved in the design, or conduct, or reporting, or dissemination plans of our research. 


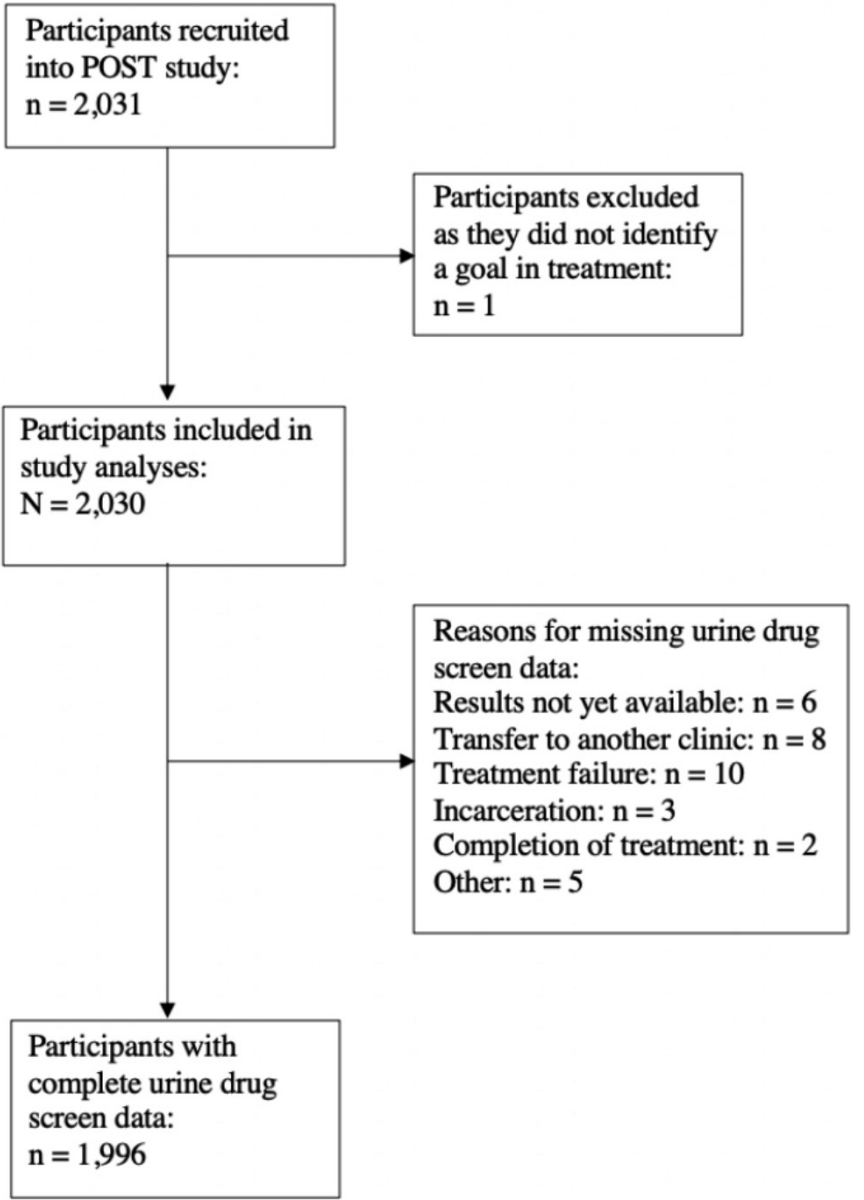

Figure 1 Study flow diagram. POST, Pharmacogenetics of Opioid Substitution Treatment Response.

\section{RESULTS}

\section{Participant characteristics and goals in treatment}

Altogether, 2030 participants were included in the analyses (figure 1; study flow diagram), with a mean age of 39.2 years $(\mathrm{SD}=10.7)$ and $44 \%$ were women (table 1$)$. The majority of participants were receiving treatment with methadone $(78.9 \%)$ compared with buprenorphinenaloxone $(21.1 \%)$ and the median length of time in treatment was 2.6 years (IQR 5.2). UDSs collected for the 3 months of study duration were available for 1996 participants. Among these participants, $57 \%$ were abstinent from opioid use during those 3 months. Ultimately, we identified six distinct 'themes' or 'goals' in treatment: (1) to control cravings or withdrawal, (2) to maintain or stabilise medication dose, (3) to stop or taper off treatment, (4) to 'stay or get clean', (5) to manage pain and (6) to 'live a normal life', as presented by our previous paper looking at patient important outcomes in the OUD population receiving opioid agonist therapy. ${ }^{22}$ The "control cravings or withdrawal' theme consisted of participants responses stating they would like to avoid withdrawal or control their cravings. Participant responses grouped in the second theme of 'to maintain or stabilise medication dose' were made up of responses indicating that they wanted to maintain opioid agonist medication doses,
Table 1 Characteristics of participants at study entry $(n=2030)$

\begin{tabular}{|c|c|}
\hline Characteristic & Statistic \\
\hline \multicolumn{2}{|l|}{ Demographic and clinical } \\
\hline Age in years; mean (SD) & $39.2(10.7)$ \\
\hline Female sex; ${ }^{*}(\%)$ & $894(44.1)$ \\
\hline \multicolumn{2}{|l|}{ Type of treatment; $n(\%)$} \\
\hline Methadone & 1601 (78.9) \\
\hline Buprenorphine-naloxone & $429(21.1)$ \\
\hline \multicolumn{2}{|l|}{ Dose in mg/day; mean (SD) } \\
\hline Methadone & $70.5(41.4)$ \\
\hline Buprenorphine-naloxone & $12.0(6.7)$ \\
\hline Years in treatment; ${ }^{*}$ median (IQR) & $2.6(5.2)$ \\
\hline Abstinence from opioid use at baseline; $†$ (\%) & 646 (31.9) \\
\hline $\begin{array}{l}\text { Number of opioid urine drug screens at } 3 \\
\text { months; } \ddagger \text { mean (SD) }\end{array}$ & $12.6(5.3)$ \\
\hline $\begin{array}{l}\text { Median percentage of opioid-positive urine drug } \\
\text { screens at } 3 \text { months; } \neq \text { median (Q1, Q3) }\end{array}$ & 0 (0 to 20$)$ \\
\hline Abstinence from opioid use at 3 months; $\ddagger n(\%)$ & $1127(56.5)$ \\
\hline \multicolumn{2}{|l|}{ Patient-reported goals in treatment§ } \\
\hline \multicolumn{2}{|l|}{ Number of goals reported; $n(\%)$} \\
\hline One & $1222(60.2 \%)$ \\
\hline Two & $643(31.7 \%)$ \\
\hline Three & $150(7.4 \%)$ \\
\hline Four & $13(0.64 \%)$ \\
\hline Five & $2(0.1 \%)$ \\
\hline Control cravings/withdrawal & $247(12.17 \%)$ \\
\hline Maintain or stabilise medication dose & $122(6.01 \%)$ \\
\hline 'Live a normal life' & $283(13.94 \%)$ \\
\hline Manage pain & $240(11.82 \%)$ \\
\hline 'Stay or get clean' & $742(36.55 \%)$ \\
\hline Stop or taper off treatment & $1386(68.28 \%)$ \\
\hline
\end{tabular}

*Data available for 2029 participants.

tData available for 2028 participants.

‡Data available for 1996 participants (missing for 34 participants).

$\S$ Percentages sum to more than $100 \%$ as patients could report

multiple goals in treatment.

Q1, 25 $5^{\text {th }}$ percentile; Q3, $75^{\text {th }}$ percentile.

stabilise their dose or did not have any reported goals. The third goal to 'stop or taper off treatment' included goals to stop treatment completely, to not be dependent on opioid agonist therapy, or to taper off or reduce their medication dose. Participant goals such as wanting to get clean, stay clean, achieve abstinence or achieve sobriety from all drugs were included in the fourth goal of 'to stay or get clean'. The fifth theme of 'pain management' either mentioned chronic pain, or pain management in general. The sixth theme of 'living a normal life' consisted of responses such as wanting a stable life, normal life, to get qualifications related to education, job or work, to achieve good mental health or wanting to support their family. ${ }^{22}$ 
The most common patient-reported goal was to 'stop or taper off treatment' (68\%; see table 1 for all goals). Other goals included to 'stay or get clean' $(37 \%)$, to 'live a normal life' (14\%) and to 'control cravings or withdrawal' (12\%). Most participants $(60.2 \%)$ reported one treatment goal (mean number of goals $=1.49, \mathrm{SD}=0.67$ ). We reported demographic and clinical characteristics associated with different treatment goals in a previously published paper. ${ }^{22}$

The proportion of participants treated with methadone (as compared with buprenorphine-naloxone) was $79 \%$ for the goal 'stop or taper off treatment', $78 \%$ for 'stay or get clean', $84 \%$ for 'live a normal life', $86 \%$ for 'manage pain', $81 \%$ for 'control cravings or withdrawal' and $75 \%$ for 'maintain or stabilise medication dose'. The median length of time in treatment at the time of study recruitment was 3 years $(\mathrm{IQR}=5)$ for the goal 'stop or taper off treatment', 2 years $(\mathrm{IQR}=4.5)$ for 'stay or get clean', 3 years $(\mathrm{IQR}=6.2)$ for 'live a normal life', 4 years $(\mathrm{IQR}=8)$ for 'manage pain', 2 years $(\mathrm{IQR}=5.4)$ for 'control cravings or withdrawal' and 5 years $(\mathrm{IQR}=8)$ for 'maintain or stabilise medication dose'. Abstinence from opioid use at study entry was observed in $33 \%$ of participants reporting the goal to 'stop or taper off treatment', $28 \%$ for 'stay or get clean', $31 \%$ for 'live a normal life', $30 \%$ for 'manage pain', $31 \%$ for 'control cravings or withdrawal' and $45 \%$ for 'maintain or stabilise medication dose'.

\section{Association between patients' goals in treatment and 3-month abstinence from opioid use}

We examined the association between patient goals and abstinence from opioid use for 3 months following study entry, adjusting for other characteristics previously shown to be associated with ongoing opioid use (table 2). Paradoxically, participants reporting the goal 'to stay or get clean' had 27\% lower odds of abstinence from opioids at
3 months $(\mathrm{OR}=0.73$, 95\% CI 0.59 to $0.91, \mathrm{p}=0.005)$, even after adjusting for baseline abstinence from opioid use. No other patient-reported goals in treatment were significantly associated with 3-month abstinence.

Good model fit was assessed using the Hosmer-Lemeshow statistic $(\chi 2=5.93, \mathrm{p}=0.656)$ and multicollinearity was not a concern (mean VIF 1.19). Using deviance residuals, we detected 14 outliers with deviance residuals greater than an absolute value of 2 . We conducted a post-hoc sensitivity analysis removing outliers and found that participants who reported the goal 'to control cravings or withdrawal' also had significantly lower odds of opioid abstinence at 3 months $(\mathrm{OR}=0.73,95 \%$ CI 0.54 to $0.99, \mathrm{p}=0.044$; online supplemental table 1). There were no other significant changes to the results upon removing outliers.

Finally, we examined the association between number of reported goals and abstinence from opioid use for 3 months (online supplemental table 2). As compared with reporting one goal in treatment, reporting two goals was not associated with opioid use $(\mathrm{OR}=0.93,95 \% \mathrm{CI}=0.75$, $1.15, \mathrm{p}=0.497$ ), however reporting three or more goals may be associated with lower odds of abstinence from opioids $(\mathrm{OR}=0.70,95 \% \mathrm{CI}=0.49,1.0, \mathrm{p}=0.049)$.

\section{DISCUSSION}

In this mixed-methods study, we examined treatment goals reported by more than 2000 patients receiving pharmacological treatment for OUD to determine their association with the frequently measured treatment outcome, opioid use. Participants reporting the goals to 'stay or get clean' and to control cravings or withdrawal were less likely to be abstinent from opioids during the next 3 months of treatment than participants who did not report those goals. Other goals related to termination of treatment, pain or

Table 2 Multivariable model of the association between patient goals and abstinence from opioid use for 3 months following study entry

\begin{tabular}{|c|c|c|c|c|c|c|}
\hline \multirow[b]{3}{*}{ Covariate } & \multicolumn{3}{|c|}{$\begin{array}{l}\text { Complete case analysis* } \\
(n=1994) \dagger\end{array}$} & \multirow{2}{*}{\multicolumn{3}{|c|}{ Sensitivity analysis excluding outliers $(n=1980)^{\star} \ddagger$}} \\
\hline & \multicolumn{3}{|l|}{1} & & & \\
\hline & OR & $95 \% \mathrm{Cl}$ & $\mathrm{p}$ value & OR & $95 \% \mathrm{Cl}$ & $\mathrm{p}$ value \\
\hline Control cravings/withdrawal & 0.76 & 0.56 to 1.03 & 0.078 & 0.73 & 0.54 to 0.99 & 0.044 \\
\hline Maintain or stabilise medication dose & 1.15 & 0.74 to 1.79 & 0.523 & 1.24 & 0.79 to 1.95 & 0.354 \\
\hline 'Live a normal life' & 1.02 & 0.77 to 1.35 & 0.879 & 0.98 & 0.74 to 1.31 & 0.902 \\
\hline Manage pain & 1.0 & 0.73 to 1.36 & 0.976 & 0.96 & 0.70 to 1.32 & 0.806 \\
\hline 'Stay or get clean' & 0.73 & 0.59 to 0.91 & 0.005 & 0.70 & 0.56 to 0.87 & 0.001 \\
\hline Stop or taper off treatment & 1.0 & 0.80 to 1.27 & 0.974 & 1.01 & 0.80 to 1.27 & 0.954 \\
\hline
\end{tabular}

Variance inflation factor $=1.19$.

Hosmer-Lemeshow $\chi^{2} 5.93, p=0.656$.

${ }^{*}$ Model is adjusted for age, sex, type of treatment (methadone or buprenorphine-naloxone), dose, length of time in treatment and opioid abstinence at baseline.

†Participants with missing data in any of the included covariates are excluded due to complete case analysis (missing urine drug screen data: $n=36$, missing sex: $n=1$, missing length of time in treatment: $n=1$ ).

‡Excluding 14 outliers detected using deviance residuals less than -2 from the analysis 
personal or social functioning were not associated with opioid use. These findings suggest that abstinence from opioids, a commonly used treatment outcome measured in clinical trials, does not reflect what patients want out of treatment, and raises questions about the alignment between treatment outcomes and patient goals. ${ }^{40}$

We found that patients who identified goals related to stopping drug use or controlling OUD symptoms had worse outcomes in treatment as measured by UDS. There is a rich literature examining the apparent contradiction between abstinence-related goals and subsequent drugtaking behaviours. This is in essence the focus of motivational interviewing ${ }^{41}$ in which clinicians help patients develop motivation through recognising discrepancies between their current situation and their goals, shifting the balance towards change. ${ }^{42}$ One possible explanation is that patients who were experiencing worse outcomes in treatment or higher severity of illness were more likely to report goals regarding management of substance use symptoms and abstinence from drug use, thus also increasing the likelihood that they experienced ongoing opioid use. Another possibility is that participants who had achieved abstinence or had improvements in OUD withdrawal symptoms may have been less likely to identify the same goals. Nonetheless, exploring why patients wishing to abstain from opioid use are not achieving this goal is an area requiring further study. Beyond quantitatively examining factors associated with ongoing substance use, previous qualitative studies that explore patient perceptions of barriers and facilitators to achieving abstinence are illuminating and may inform future interventions and research. ${ }^{43-45}$

Although the majority of patients wanted to taper off or stop treatment, this goal had no association with abstinence from opioid use. One possible explanation is that participants may have been unhappy with treatment and therefore non-adherent. Factors associated with nonadherence to opioid agonist treatments have been previously studied. ${ }^{46-49}$ There is a vast literature on factors affecting patient adherence to treatment in general and, notably, no single explanation sufficiently accounts for variation in adherence. ${ }^{50}$ Authors in this field have suggested considering the patient's experience of illness and its meaning as important factors to study in understanding adherence to treatment. ${ }^{5051}$ This finding calls into question the rationale for entering and continuing pharmacological treatment while continuing to use opioids for this group of patients. Furthermore, this is a particularly important finding, given that retention in treatment is among the most consistently measured outcomes, ${ }^{19}$ and guidance around taper and discontinuation of long-term opioid agonist treatments for OUD is limited. ${ }^{452}$ Studies examining opioid agonist tapers have identified challenges and risks of poor outcomes ${ }^{53} 54$ including withdrawal symptoms, return to drug use, pain, psychiatric symptoms, hospitalisation and death. ${ }^{556}$ A previous study found that patients' interest in stopping treatment was associated with shorter duration of treatment and lack of concern about relapse to opioid use. ${ }^{57}$ This is concerning as one would hope patients planning to stop treatment would be reliably abstinent from opioids. What distinguishes this group of patients who wish to discontinue treatment? Whether some of these patients are mandated to be in treatment is unknown. Better understanding patients' reasons for wanting to stop or taper treatment and examining outcomes for patients who initiate an opioid agonist taper is imperative.

Other patient identified goals in treatment that were not associated with the results of their UDS, included goals around pain management, and the goal 'to live a normal life'. This suggests that clinicians and researchers may require additional tools to measure outcomes related to those patient-important treatment goals. Tools validated to assess pain in this population include the Brief Pain Inventory ${ }^{58}$ and social functioning may be examined using the Maudsley Addiction Profile. ${ }^{60} \mathrm{~A}$ more nuanced understanding of specific goals around personal and social functioning, on a population and individual level, is required in order to be able to appropriately assess and address these goals during treatment. Overall, our finding that results of UDSs are not associated with all patient goals in treatment is expected as UDS results would not be expected to be a proxy for all of the different goals. However, this study adds evidence to the notion that traditional metrics of success in opioid use disorder treatment are insufficient in isolation. It is important to note that although patient goals appear to have limited predictive value on opioid use during treatment, this does not imply that clinicians should not ask patients about their treatment goals. It is not uncommon that patients have goals that are not achieved in treatment (eg, weight loss, increased physical activity) and this does not mean that clinicians or patients should give up on these goals or should not enquire about them. Rather, we must consider how well traditional metrics of treatment success align with desired treatment outcomes for all stakeholders, especially patients, and consider additional ways to evaluate and improve treatment success based on patients' self-reported goals.

Finally, in a previous paper, we examined group differences between participants selecting each treatment goal. ${ }^{22}$ Women were more likely to report the goal of stopping treatment. Older age, first exposure to opioids through physician prescription and unemployment were all associated with greater odds of reporting goals related to pain management. ${ }^{22}$ These findings indicated that, unsurprisingly, patients' characteristics are associated with their treatment goals and may help to guide focussed questioning and evaluation of patients' goals in treatment.

This study has a number of potential limitations. First, this study interprets and summarises the patients' narrative when expressing their goals in treatment using qualitative methods; however, this interpretation carries limitations related to the potential influence of social desirability bias and the influence of contextual factors on patients' responses that have not been explored in this 
study. Though beyond the scope of this paper, sociological approaches to qualitative analysis include critical appraisal of the circumstances of the participant and the context in which statements are expressed. Furthermore, there may be a healthy user/volunteer bias, ${ }^{61}$ such that individuals with better outcomes in treatment may have been more likely to participate. Additionally, the goals and treatment outcomes of patients newly entering treatment may differ from those of patients who have been in treatment longer. Patients who may have successfully achieved their goal of treatment termination were not captured by this study since they would no longer be on OUD thus not recruited. The findings in this study may not generalise to settings in which opioid agonist medications take on a primarily abstinencebased role in treatment. In Canada, pharmacological treatment for OUD is provided largely in a harm-reduction model, in which retention in treatment is not contingent on abstinence from opioids or non-opioid substances. This study did not measure patient's satisfaction or perception of treatment success or perception of meeting their goals. Future studies that examine patient satisfaction in treatment may wish to determine whether perception of treatment success correlates with programme-measured outcomes such as opioid abstinence.

\section{CONCLUSION}

Patients report a number of different goals in their treatment for OUD, which are not associated with traditional goals of treatment programmes and outcomes measured in clinical settings (abstinence from opioid use measured by UDS). We found that patients who identified goals related to stopping drug use or controlling OUD symptoms were more likely to have ongoing opioid use. However, goals unrelated to drug use carried no significant association with opioid use status. Patients reporting the goal of wanting to stop treatment were no more likely to be abstinent from opioids. The patient-identified goals to manage pain or 'live a normal life' had no association with ongoing opioid use. Future studies are needed to examine outcomes related to the goals in treatment identified in our study. Are these goals being met in treatment? For example, do patients feel their pain is well managed? Do they achieve employment? Can they achieve the goal of stopping treatment without adverse consequences? As core outcome sets are developed, patient-important outcomes remain essential to consider and may help with implementing patient-centred approaches to treatment.

\footnotetext{
Author affiliations

${ }^{1}$ Department of Psychiatry and Behavioral Neurosciences, McMaster University, Hamilton, Ontario, Canada

${ }^{2}$ Department of Health Research, Evidence and Impact, McMaster University, Hamilton, Ontario, Canada

${ }^{3}$ Department of Family Medicine, McMaster University, Hamilton, Ontario, Canada ${ }^{4}$ Neurosciences Graduate Program, McMaster University, Hamilton, Ontario, Canada ${ }^{5}$ Michael G. DeGroote School of Medicine, McMaster University, Hamilton, Ontario, Canada

${ }^{6}$ Medical Science Gradaute Program, McMaster University, Hamilton, Ontario, Canada
}

${ }^{7}$ Department of Medicine, McMaster University, Hamilton, Ontario, Canada

${ }^{8}$ Northern Ontario School of Medicine, Sudbury, Ontario, Canada

${ }^{9}$ Canadian Addiction Treatment Centres, Markham, Ontario, Canada

${ }^{10}$ ICES North, Sudbury, Ontario, Canada

${ }^{11}$ Health Sciences North Research Institute, Sudbury, Ontario, Canada

${ }^{12}$ Department of Family Practice, University of British Columbia, Vancouver, British Columbia, Canada

${ }^{13}$ Biostatistics Unit, Research Institute at St Joseph's Healthcare, Hamilton, Ontario, Canada

Correction notice This article has been corrected since it first published. The provenance and peer review statement has been included.

Acknowledgements We would like to thank the Pharmacogenetics of Opioid Substitution Treatment Response (POST) study participants for their time and contributions, without which this study would not be possible.

Contributors TR, LN, BP, NS, BD and ZS are responsible for the study concept and design. TR, BP, LT and ZS developed the methods and data analysis. TR conducted quantitative analysis and $\mathrm{BP}$ conducted qualitative analysis. TR wrote the first draft of the manuscript, and TR, LN, BP, DC, NS, BD, DM, LR, AW, LT and ZS, contributed to writing and critically revising the final manuscript. All authors reviewed and approved the final manuscript.

Funding This study was supported by research grants from the Canadian Institutes for Health Research (grant numbers PJT-156306 and SHI-155404).

Competing interests Dr David C. Marsh reports Salary income as Chief Medical Director, Canadian Addiction Treatment Centres and as Associate Dean Research, Innovation and International Relations, Northern Ontario School of Medicine.

Patient consent for publication Not required.

Ethics approval This study was reviewed and approved by the Hamilton Integrated Research Ethics Board (project ID 4556) and conducted in accordance with its ethical guidelines.

Provenance and peer review Not commissioned; externally peer reviewed.

Data availability statement Data are available upon reasonable request. The data sets used during the current study are available from the corresponding author on reasonable request.

Supplemental material This content has been supplied by the author(s). It has not been vetted by BMJ Publishing Group Limited (BMJ) and may not have been peer-reviewed. Any opinions or recommendations discussed are solely those of the author(s) and are not endorsed by BMJ. BMJ disclaims all liability and responsibility arising from any reliance placed on the content. Where the content includes any translated material, BMJ does not warrant the accuracy and reliability of the translations (including but not limited to local regulations, clinical guidelines, terminology, drug names and drug dosages), and is not responsible for any error and/or omissions arising from translation and adaptation or otherwise.

Open access This is an open access article distributed in accordance with the Creative Commons Attribution Non Commercial (CC BY-NC 4.0) license, which permits others to distribute, remix, adapt, build upon this work non-commercially, and license their derivative works on different terms, provided the original work is properly cited, appropriate credit is given, any changes made indicated, and the use is non-commercial. See: http://creativecommons.org/licenses/by-nc/4.0/.

\section{ORCID iDs}

Tea Rosic http://orcid.org/0000-0001-7406-4056

Leen Naji http://orcid.org/0000-0003-0994-1109

Nitika Sanger http://orcid.org/0000-0002-5883-1873

Zainab Samaan http://orcid.org/0000-0002-5974-9361

\section{REFERENCES}

1 Special Advisory Committee on the Epidemic of Opioid Overdoses. National report: apparent opioid-related deaths in Canada (January 2016 to December 2018). web based report. Ottawa: Public Health Agency of Canada, 2019.

2 Fischer B, Kurdyak P, Goldner E, et al. Treatment of prescription opioid disorders in Canada: looking at the 'other epidemic'? Subst Abuse Treat Prev Policy 2016;11:12. 
3 Ontario Drug Policy Research Network. Ontario prescription opioid tool. Toronto, ON, 2018.

4 Bruneau J, Ahamad K, Goyer Marie-Ėve, et al. Management of opioid use disorders: a national clinical practice guideline. Can Med Assoc J 2018;190:E247-57

5 Mattick RP, Breen C, Kimber J. Methadone maintenance therapy versus no opioid replacement therapy for opioid dependence. Cochrane Database Syst Rev 2009;3:CD002209.

6 Nielsen S, Larance B, Degenhardt L, et al. Opioid agonist treatment for pharmaceutical opioid dependent people. Cochrane Database Syst Rev 2016;5:CD011117.

7 Dennis BB, Naji L, Bawor M, et al. The effectiveness of opioid substitution treatments for patients with opioid dependence: a systematic review and multiple treatment comparison protocol. Syst Rev 2014;3:105.

8 Li Y, Kantelip J-P, Schieveen PG-van, et al. Interindividual variability of methadone response. Mol Diagn Ther 2008;12:109-24.

9 Huang CL-C, Lee CW. Factors associated with mortality among heroin users after seeking treatment with methadone: a population-based cohort study in Taiwan. J Subst Abuse Treat 2013;44:295-300.

10 Zador D, Sunjic S. Deaths in methadone maintenance treatment in New South Wales, Australia 1990-1995. Addiction 2000;95:77-84.

11 Ferri M, Davoli M, Perucci CA. Heroin maintenance for chronic heroin-dependent individuals. Cochrane Database Syst Rev 2011;12:CD003410.

12 Deshpande PR, Rajan S, Sudeepthi BL, et al. Patient-Reported outcomes: a new era in clinical research. Perspect Clin Res 2011;2:137-44.

13 Marchand K, Beaumont S, Westfall J, et al. Patient-Centred care for addiction treatment: a scoping review protocol. BMJ Open 2018;8:e024588.

14 Kolind T, Hesse M. Patient-Centred care-perhaps the future of substance abuse treatment. Addiction 2017;112:465-6.

15 National Research Council. Crossing the quality chasm: a new health system for the 21st century. Washington, DC: National Academies Press, 2001.

16 Barry MJ, Edgman-Levitan S. Shared decision making--pinnacle of patient-centered care. N Engl J Med 2012;366:780-1.

17 Stewart M, Brown JB, Donner A, et al. The impact of patientcentered care on outcomes. J Fam Pract 2000;49:796-804

18 Marchand K, Beaumont S, Westfall J, et al. Conceptualizing patient-centered care for substance use disorder treatment: findings from a systematic scoping review. Subst Abuse Treat Prev Policy 2019;14:37

19 International Consortium for Health Outcomes Measurement. ICHOM standard set for addiction. Available: https://www.ichom.org/ portfolio/addiction/ [Accessed 26 Oct 2020].

20 Wiessing L, Ferri M, Darke S, et al. Large variation in measures used to assess outcomes of opioid dependence treatment: a systematic review of longitudinal observational studies. Drug Alcohol Rev 2018;37 Suppl 1:S323-38

21 Dennis BB, Sanger N, Bawor M, et al. A call for consensus in defining efficacy in clinical trials for opioid addiction: combined results from a systematic review and qualitative study in patients receiving pharmacological assisted therapy for opioid use disorder. Trials 2020;21:30.

22 Sanger N, Panesar B, Rosic T, et al. The future of precision medicine in opioid use disorder: inclusion of patient-important outcomes in clinical trials. Braz J Psychiatry 2020;00. doi:10.1590/1516-44462019-0734. [Epub ahead of print: 12 Jun 2020].

23 American Psychiatric Association. Diagnostic and statistical manual of mental disorders. 5th edn. Arlington, VA: American Psychiatric Publishing, 2013

24 Plano Clark VL, Huddleston-Casas CA, Churchill SL, et al. Mixed methods approaches in family science research. J Fam Issues 2008;29:1543-66.

25 Doyle L, Brady A-M, Byrne G. An overview of mixed methods research. Nurs Res 2009;14:175-85.

26 Samaan Z, Bawor M, Dennis BB, et al. Genetic influence on methadone treatment outcomes in patients undergoing methadone maintenance treatment for opioid addiction: a pilot study. Neuropsychiatr Dis Treat 2014;10:1503-8.

27 FaStep Assay. Trimedic supply network LTD. Available: https://www. trimedic-inc.com/wp-content/uploads/2018/04/Fastep-PackageInsert.pdf

28 Donovan DM, Bigelow GE, Brigham GS, et al. Primary outcome indices in illicit drug dependence treatment research: systematic approach to selection and measurement of drug use end-points in clinical trials. Addiction 2012;107:694-708.
29 von Elm E, Altman DG, Egger M, et al. Strengthening the reporting of observational studies in epidemiology (STROBE) statement: guidelines for reporting observational studies. BMJ 2007;335:806-8.

30 Braun V, Clarke V. Using thematic analysis in psychology. Qual Res Psychol 2006;3:77-101.

31 QSR International Pty Ltd. NVivo qualitative data analysis software. Version 12, 2018

32 Guest G, MacQueen KM, Namey EE. Applied thematic analysis. Thousand Oaks, CA: SAGE Publications, Inc, 2012.

33 O'Brien BC, Harris IB, Beckman TJ. Standards for reporting qualitative research: a synthesis of recommendations. Acad Med 2014;89:1245-51.

34 Bawor M, Dennis BB, Bhalerao A, et al. Sex differences in outcomes of methadone maintenance treatment for opioid use disorder: a systematic review and meta-analysis. CMAJ Open 2015;3:E344-51.

35 Bawor M, Dennis BB, Varenbut M, et al. Sex differences in substance use, health, and social functioning among opioid users receiving methadone treatment: a multicenter cohort study. Biol Sex Differ 2015;6:21.

36 Strain EC, Bigelow GE, Liebson IA, et al. Moderate- vs high-dose methadone in the treatment of opioid dependence: a randomized trial. JAMA 1999;281:1000-5

37 Eastwood B, Strang J, Marsden J. Effectiveness of treatment for opioid use disorder: a national, five-year, prospective, observational study in England. Drug Alcohol Depend 2017;176:139-47.

38 Kelly SM, O'Grady KE, Brown BS, et al. The role of patient satisfaction in methadone treatment. Am J Drug Alcohol Abuse 2010;36:150-4.

39 Peduzzi P, Concato J, Kemper E, et al. A simulation study of the number of events per variable in logistic regression analysis. $J$ Clin Epidemiol 1996:49:1373-9.

40 Liberati A. Need to re-align patient-oriented and commercial and academic research. Cochrane Database Syst Rev 2011;12:ED000094.

41 Miller WR, Rose GS. Toward a theory of motivational interviewing Am Psychol 2009;64:527-37.

42 Westra HA, Aviram A. Core skills in motivational interviewing. Psychotherapy 2013;50:273-8.

43 Herbeck DM, Brecht M-L, Christou D, et al. A qualitative study of methamphetamine users' perspectives on barriers and facilitators of drug abstinence. J Psychoactive Drugs 2014:46:215-25.

44 Moran L, Keenan E, Elmusharaf K. Barriers to progressing through a methadone maintenance treatment programme: perspectives of the clients in the Mid-West of Ireland's drug and alcohol services. BMC Health Serv Res 2018;18:911.

45 Notley C, Blyth A, Maskrey V, et al. The experience of long-term opiate maintenance treatment and reported barriers to recovery: a qualitative systematic review. Eur Addict Res 2013;19:287-98.

46 Roux P, Lions C, Michel L, et al. Predictors of non-adherence to methadone maintenance treatment in opioid-dependent individuals: implications for clinicians. Curr Pharm Des 2014;20:4097-105.

47 Tran BX, Nguyen LH, Tran TT, et al. Social and structural barriers for adherence to methadone maintenance treatment among Vietnamese opioid dependence patients. PLoS One 2018:13:e0190941.

48 Fareed $A$, Eilender $P$, Ketchen $B$, et al. Factors affecting noncompliance with buprenorphine maintenance treatment. J Addict Med 2014:8:345-50.

49 Launonen E, Wallace I, Kotovirta E, et al. Factors associated with non-adherence and misuse of opioid maintenance treatment medications and intoxicating drugs among Finnish maintenance treatment patients. Drug Alcohol Depend 2016;162:227-35.

50 Vermeire E, Hearnshaw H, Van Royen P, et al. Patient adherence to treatment: three decades of research. A comprehensive review. J Clin Pharm Ther 2001;26:331-42.

51 Conrad P. The meaning of medications: another look at compliance. Soc Sci Med 1985;20:29-37.

52 College of Physicians and Surgeons of British Columbia. Methadone maintenance program: clinical practice guidelines, 2015. Available: http://www.bccdc.ca/resource-gallery/Documents/Statistics\% 20and\%20Research/Publications/Epid/Other/02 CPSBCMethadone_Maintenance_Program_Clinical\%20_Practice_Guideline. pdf [Accessed 11 Apr 2020].

53 Magura S, Rosenblum A. Leaving methadone treatment: lessons learned, lessons forgotten, lessons ignored. Mt Sinai J Med 2001;68:62-74.

54 Latowsky M. Improving detoxification outcomes from methadone maintenance treatment: the interrelationship of affective states and protracted withdrawal. J Psychoactive Drugs 1996;28:251-7.

55 Calsyn DA, Malcy JA, Saxon AJ. Slow tapering from methadone maintenance in a program encouraging indefinite maintenance. $J$ Subst Abuse Treat 2006;30:159-63. 
56 Nosyk B, Sun H, Evans E, et al. Defining dosing pattern characteristics of successful tapers following methadone maintenance treatment: results from a population-based retrospective cohort study. Addiction 2012;107:1621-9.

57 Winstock AR, Lintzeris N, Lea T. "Should I stay or should I go?" Coming off methadone and buprenorphine treatment. Int J Drug Policy 2011;22:77-81.

58 Cleeland C. The brief pain inventory: user guide. Texas, USA, 1991.
59 Dennis BB, Roshanov PS, Bawor M. Usefulness of the brief pain inventory in patients with opioid addiction receiving methadone maintenance treatment. Pain Physician 2016;19:E181-95.

60 Marsden J, Gossop M, Stewart D, et al. The Maudsley addiction profile (MAP): a brief instrument for assessing treatment outcome. Addiction 1998;93:1857-67.

61 Shrank WH, Patrick AR, Brookhart MA. Healthy user and related biases in observational studies of preventive interventions: a primer for physicians. J Gen Intern Med 2011;26:546-50. 